\title{
The Application of GPGPU to Automatic Electron Gun Alignment in the Scanning Electron Microscope
}

\author{
D. M. Holburn ${ }^{1}$, B.C. Breton ${ }^{1}, \mathrm{An} \mathrm{Li}^{1}$ and N. H. M. Caldwell ${ }^{2}$ \\ 1. Department of Engineering, University of Cambridge, Trumpington Street, Cambridge, CB2 1PZ, \\ United Kingdom \\ 2. Suffolk Business School, University Campus Suffolk, Waterfront Building, Neptune Quay, Ipswich, \\ IP4 1QJ, United Kingdom
}

Once trained, the human brain is an excellent image processor; to replicate that skill for scanning electron microscope (SEM) image assessment demands considerable computing power. We have evaluated parallel computing for this purpose and developed an image processing tool for the automatic optimisation of electron gun alignment. The instrumental setup for this work was a Carl Zeiss 1430VP SEM with tungsten thermal-emission firing unit.

Although SEM electron guns vary from manufacturer to manufacturer, careful alignment of the gun is an essential step in obtaining the best possible image, and aims to achieve perfect coaxial alignment of the electron beam path with the electron-optical axis. In most tungsten-based systems this is accomplished first by careful attention to mechanical positioning during the installation of the firing unit. However, mechanical adjustment alone is not sufficient to ensure optimum alignment. In the $1430 \mathrm{VP}$ a system of magnetic alignment coils is provided to give fine control of the beam's trajectory down the column. These are configured as upper and lower pairs of orthogonal coils that can be driven so as to allow two-dimensional control of gun shift and gun tilt. An emission-image mode is provided in which an image of the gun cross-over is scanned across an aperture in the optical path to help the operator make appropriate adjustments. Manual alignment is an iterative process calling for many precise adjustments to be made to the four individual coils, and requires some experience to carry out. Gun alignment may need to be performed on a regular basis: to compensate for filament drift, or whenever changes are made to the imaging conditions - for example, change of accelerating voltage or working distance. For these reasons, a robust method was sought to obtain fast and accurate gun alignment based on assessment of the emission image.

GPGPU (General Purpose computation on Graphics Processing Units) is now an established means of exploiting the parallel computational power available in the tens, hundreds or even thousands of processor cores on certain graphics cards. Recent software advances have greatly facilitated the formulation of a range of mathematical problems into parallel form. A powerful example of this is the Compute Unified Device Architecture (CUDA) introduced by NVIDIA in 2006, which has enabled developers to utilise GPUs by means of extensions to standard programming languages.

While GPUs may not process data items as fast as high-performance CPUs, GPUs process many items in parallel as opposed to one at a time as in CPUs. This vastly higher GPU throughput offers the potential for orders of magnitude speed-up in the overall computation. Use of GPGPU is therefore most advantageous where the same piece of code (or kernel) needs to be executed on large data arrays. This scenario is encountered regularly with SEMs and other microscopes generating large digital images, since such images are in essence large two-dimensional arrays. 
As part of our continuing research programme into improving SEM technologies, we have undertaken several projects to investigate the potential of GPGPU for the SEM. In earlier projects we explored the application of CUDA in automatic focusing [1] and in high-speed arithmetic image processing and analysis [2]. Microscope control can also benefit substantially from GPGPU's enhanced computational throughput, as many microscope control functions may be formulated as operations of image processing and analysis, with instrumental sub-systems being adjusted on an automated basis so as to optimise the characteristics of acquired images, which may correspond to the normal display image, or to some other data set. In this work, the objective was to automate the alignment of the SEM electron gun.

A NVIDIA GeForce GTX570 graphics card with 480 processor cores was used. Programming was undertaken using Carl Zeiss' Application Programming Interface to the SmartSEM software, which meant that external programs could directly access and control instrument parameters locally or remotely, across a network. Parts of the code were prototyped and run on stand-alone workstations equipped with a range of GPUs of differing specifications. The feature of remote access to the SEM facilitated comparison of the effectiveness of a number of different GPUs for this work. The software was designed and implemented using a combination of the CUDA Toolkit, the NVIDIA GPU Computing SDK, and Microsoft Visual Studio.

The automated method developed resembles the procedure adopted by an experienced operator. The emission image is of a unique form, typically showing a low-contrast image of the gun aperture and, within this, a characteristic shape representing the image of the crossover, dependent on filament current and other parameters. For best alignment, both need to be centred in the field of view. This is achieved through the application of median filtering, maximum filtering and minimum filtering, to locate the image of the aperture, and analysis of white-dot density in the crossover image to locate its centroid. With these identified, suitable coil currents can be calculated to allow the aperture and the crossover to be centred. This requires a calibration procedure that need be carried out only once for the target microscope. All the key image processing operations benefit substantially from GPGPU assistance, e.g. with a full-screen image, median filtering executes 30x faster in the GTX570 than in the Intel CPU (i7-960); other kinds of filtering are similarly enhanced. Once the system has been initialized, a full alignment process can be carried out within 2 seconds, including adjustment of brightness and contrast.

These results provide further confirmation that GPGPU techniques have the potential to produce dramatic improvements in instrumental performance and convenience in scanning electron microscopy. More recent experiments suggest that a similar approach can be applied successfully to achieve automated alignment of other kinds of electron source [3].

\section{References}

[1] N.H.M. Caldwell et al, Microsc. Microanal. 19 (Suppl S2) (2013), p. 792.

[2] N.H.M. Caldwell et al, Microsc. Microanal. 19 (Suppl S2) (2013), p. 774.

[3] NVIDIA GeForce and CUDA are trademarks of NVIDIA Corporation. SmartSEM ${ }^{\mathrm{TM}}$ is a trademark of Carl Zeiss Microscopy $\mathrm{GmbH}$. This research was supported by funding from Carl Zeiss Microscopy. The authors gratefully acknowledge the assistance of Carl Zeiss staff, especially Daniel Aldridge, David Hubbard and Stewart Bean. 